\title{
Solute transport in partially-saturated deformable porous media: Application to a landfill clay liner
}

\author{
H J Zhang ${ }^{1}$, D-S Jeng ${ }^{1, \#}$, B R Seymour ${ }^{2}$, D A Barry ${ }^{3}, \mathrm{~L} \mathrm{Li}^{4}$ \\ ${ }^{1}$ Division of Civil Engineering, University of Dundee, DD1 4HN, UK \\ ${ }^{2}$ Department of Mathematics, University of British Columbia, Vancouver BC, Canada V6Z $1 Z 2$ \\ ${ }^{3}$ Laboratoire de technologie écologique, Faculté del'environnement naturel, architectural et \\ construit (ENAC), Station 2, Ecole Polytechnique Fédérale de Lausanne (EPFL), 1015 Lausanne, \\ Switzerland \\ ${ }^{4}$ School of Engineering, University of Queensland, Queensland, Australia \\ \# Corresponding author, email: d.jeng@dundee.ac.uk
}

\begin{abstract}
Based on the one-dimensional Biot consolidation equations, this paper develope$\mathrm{d}$ an advection-diffusion equation that incorporates saturation, compressibility of the pore fluid and longitudinal dispersivity of the solute transport in an unsaturated, deforming porous medium. A simplified model was proposed for the case of a landfill liner. Numerical results demonstrated that the longitudinal dispersivity and compressibility of the pore fluid can be significant. Furthermore, the degree of soil saturation and loading rate of the waste surcharge affect significantly the contamination advective emission, namely the cumulative contaminant mass outflow per unit area from compacted clay liner (CCL) due to advective flow.

Keywords: Biot consolidation, advection-diffusion, pore water compressibility
\end{abstract}

\section{Introduction}

Various environmental situations are typically investigated using solutions of the solute transport equations considering the porous medium to be rigid (e.g., 
$[1,2,3,4,5])$. In such cases, no volume change occurs during the transport process and therefore the advection is determined solely by the hydraulic gradient. However, porous medium deformations can lead to unsteady advective flow. Some examples include solute transport through a clay liner during waste-filling operations, dredged contaminated sediment after placement in a confined disposal facility, consolidation of contaminated sediments due to overburden of capping material, and solute transport in cartilage under mechanical load (e.g., [6, 7, 8]). In these cases, the deformation and solute transport processes occur simultaneously and coupled effects should be considered.

Modeling of contaminant transport through deformable porous media has received attention during the last two decades. Potter and Gibson [9] presented a model for dissolved phase advection-dispersion transport using Terzaghi consolidation theory. Smith [6] derived a one-dimensional theory of contaminant migration based on a small strain analysis of a consolidating soil. The equations were recast in a material coordinate system for problems involving large deformation or a moving boundary. Peters and Smith [10] extended the previous model of Smith [6] for transient solute transport within a deformable porous medium for both small and large deformations. Moo-Young et al.[11] presented experimental results of contaminant transport in soil specimens undergoing consolidation induced by a centrifuge; consolidation was observed to accelerate solute migration. With the coupled Terzaghi consolidation and ADE (advection-diffusion equation) equation, Alshawabkeh et al.[12] calculated the contaminant mass flux that was enhanced by the capping load-induced sediment consolidation. They concluded that advection caused by consolidation will accelerate the breakthrough of the contaminant through the cap [12]. Arega and Hayter [7] used a one-dimensional 
large strain consolidation and contaminant transport model to simulate capping consolidating contaminated sediment based on reduced coordinates.

In addition to the approaches for small strain, Lewis [13] generalized the finite strain consolidation and solute transport model of Peters and Smith [10] by incorporating self-weight in the consolidation process, and included more general constitutive functions for consolidation and transport coefficients. Fox and co-workers $[14,15,16]$ adopted a piecewise linear approach to handle coupled one-dimensional large strain consolidation and two-dimensional solute transport in a confined disposal facility for dredged contaminated sediments.

In real environments, unsaturated porous media are common $[17,18,19]$. For example, marine sediments are often unsaturated due to gas produced in biochemical processes. Another case is where the groundwater table is located some distance below a landfill geomembrane, in which case the soil beneath the landfill will be partially saturated [17].

In this study, the case of a landfill liner is considered, for which a one-dimensional Biot consolidation equation is used to describe flow in an unsaturated porous medium incorporating the self-weight of the liner. The situation considered is that of compressible pore water at a fixed saturation. The ADE that is typically used to describe solute transport through a rigid porous medium [1] is modified to include partial saturation, CPW (compressible pore water), SVP (spatial variation of porosity) and longitudinal dispersivity. The equations are non-dimensionalized, identifying nine important parameters. The importance of these parameters is discussed for a range of physical conditions. A hypothetical engineered landfill liner is used as an illustrative example, demonstrating the influence of partial saturation and the loading process on contaminant migration. 


\section{Theoretical Formulation}

\subsection{Consolidation equation}

Here we state the basic equations linking flow velocity with excess pore pressure. The one-dimensional unsaturated fluid storage (Appendix) and Biot equations [20] are, respectively,

$$
\begin{gathered}
S_{r} n \beta \frac{\partial p^{e}}{\partial t}+S_{r} \frac{\partial^{2} u}{\partial t \partial z}=\frac{1}{\rho_{w} g} \frac{\partial}{\partial z}\left(K \frac{\partial p^{e}}{\partial z}\right) \\
G \frac{2(1-v)}{(1-2 v)} \frac{\partial^{2} u}{\partial z^{2}}+\left(1-n^{0}\right)\left(\rho_{s}-\rho_{w}\right) g \frac{\partial u}{\partial z}=\frac{\partial p^{e}}{\partial z} .
\end{gathered}
$$

where $p^{e}$ is excess pore pressure, $u$ is soil displacement. $S_{r}, n, n^{0}, K, G$ and $v$ represent degree of saturation, current porosity, initial porosity, hydraulic conductivity, shear modulus and Poisson's ratio, respectively; $\rho_{w}, \rho_{s}$ are the density of pore water and solid materials, respectively. Note that the compressive effective normal stress is negative.

redIn this study, density of both components of soil are independent of the dilute solute concentration [21]. When the sorption occurs, the mass of a unit volume of solid grains (i.e., density) $\rho_{s}$ becomes $\rho_{s}\left(1+K_{d} c_{f}\right)$. Using the clay liner as an example, the measured VOC concentration in the landfill leachate ranges from 10 to $10^{4} \mu \mathrm{g} / \mathrm{l}$ [21]. Lewis et al. [22] adopted the distribution coefficient $K_{d}=1 \mathrm{mg} / \mathrm{l}$, leading to the change of the density of solid due to sorption is less than $0.001 \%$, which is negligible. Consequently, it is reasonable to assume that $\rho_{s}$ is independent of the solute mass concentration. Therefore, the assumption of volume-preserving deformation of the solid phase embedded in derivation (Appendix) can be ensured, i.e., $\nabla \bullet \vec{v}_{s}=0$ [23]. 
The compressibility of pore fluid in clay, $\beta$, depends on the degree of saturation $S_{r}$, the amount of dissolved air in pore water and absolute air pressure. It can be estimated by [24]

$$
\beta=\frac{S_{r}}{K_{w 0}}+\frac{1-S_{r}+r_{h} S_{r}}{P_{a}+P_{0}}
$$

where $K_{w 0}$ is the pore water bulk modulus, $r_{h}$ denotes volumetric fraction of dissolved air within pore water, $P_{a}$ denotes gauge air pressure and $P_{0}$ represents the atmosphere pressure. In the high saturation limit, when $r_{h}=0.02, S_{r}=0.8 \sim 1.0$ and $\beta$ falls into the range of $2 \times 10^{-6} \sim 2 \times 10^{-7} \mathrm{~Pa}^{-1}$.

\subsection{Solute transport equation}

Following [10], the solute transport equation in a one-dimensional deforming porous medium is

$$
\frac{\partial\left(n S_{r} c_{f}\right)}{\partial t}+\frac{\partial\left[(1-n) c_{s}\right]}{\partial t}=-\frac{\partial}{\partial z}\left[n S_{r}\left(-D \frac{\partial c_{f}}{\partial z}+v_{f} c_{f}\right)+(1-n) v_{s} c_{s}\right],
$$

where $c_{f}$ and $c_{s}$ are the concentration of the solute in the fluid and solid phase, respectively; $D$, which represents the hydrodynamic dispersion coefficient, is the sum of the effective molecular diffusion, $D_{m}$, and mechanical dispersion, $\alpha_{L}\left(v_{f}-\right.$ $v_{s}$ ), where $v_{f}$ denotes the average fluid velocity and $v_{s}$ is the velocity of the solid. redHere, the effective molecular diffusion tensor, mechanical dispersion tensor and consequently the hydrodynamic dispersion tensor reduce to scalar for the onedimensional isotropic soil.

By definition the concentration of the contaminant in the solid phase is

$$
c_{s}=\rho_{s} S=\rho_{s} F\left(c_{f}, t\right),
$$


where $S$ is the mass of contaminant sorbed onto the solid phase per unit mass of solid phase, and $F$ is a function describing the relationship. If sorption is an equilibrium linear reversible process, then [6]

$$
S=K_{d} c_{f},
$$

where $K_{d}$ describes the partitioning of the contaminant.

Based on the mass balance equations for the fluid, Eq. (34), and solid phases, Eq. (41) (introduced in the Appendix), and considering the solid particles as incompressible (i.e., $\rho_{s}$ is constant), Eq. (4) becomes

$$
\begin{aligned}
S_{r} n \frac{\partial c_{f}}{\partial t}+(1-n) \frac{\partial c_{s}}{\partial t}= & \frac{\partial}{\partial z}\left(S_{r} n D \frac{\partial c_{f}}{\partial z}\right)-S_{r} n v_{f} \frac{\partial c_{f}}{\partial z}-(1-n) v_{s} \frac{\partial c_{s}}{\partial z} \\
& +S_{r} n \beta\left(\frac{\partial p^{e}}{\partial t}+v_{f} \frac{\partial p^{e}}{\partial z}\right) c_{f} .
\end{aligned}
$$

Assuming the linear sorption relationship described by Eqs. (5) and (6), and using Eq. (56) in the Appendix, Eq. (7) becomes

$$
\begin{aligned}
{\left[S_{r} n+(1-n) \rho_{s} K_{d}\right] \frac{\partial c_{f}}{\partial t} } & =S_{r} n D_{m} \frac{\partial^{2} c_{f}}{\partial z^{2}}-\alpha_{L} \frac{K}{\rho_{w} g} \frac{\partial p^{e}}{\partial z} \frac{\partial^{2} c_{f}}{\partial z^{2}} \\
& +\frac{\partial c_{f}}{\partial z}\left\{-\alpha_{L} S_{r} n \beta \frac{\partial p^{e}}{\partial t}-\alpha_{L} S_{r} \frac{\partial^{2} u}{\partial z \partial t}\right. \\
& +\frac{\alpha_{L} \beta K}{\rho_{w} g}\left(\frac{\partial p}{\partial z}\right)^{2}+S_{r} D_{m} \frac{(1-n)^{2}}{1-n^{0}} \frac{\partial^{2} u}{\partial z^{2}} \\
& \left.+\frac{K}{\rho_{w} g} \frac{\partial p^{e}}{\partial z}-\left[S_{r} n+(1-n) \rho_{s} K_{d}\right] \frac{\partial u}{\partial t}\right\} \\
& +S_{r} n \beta \frac{\partial p^{e}}{\partial t} c_{f}-\beta \frac{K}{\rho_{w} g}\left(\frac{\partial p^{e}}{\partial z}\right)^{2} c_{f}+S_{r} n \beta \frac{\partial u}{\partial t} \frac{\partial p^{e}}{\partial z} c_{f} .
\end{aligned}
$$

Details of the derivation are given in the Appendix. 
It is worthwhile to compare the transport equation proposed by Peters and Smith [10] (Eq. (30) in their paper) with that given here. In the present notation, their result is

$$
n \frac{\partial c_{f}}{\partial t}+(1-n) \frac{\partial c_{s}}{\partial t}=\frac{\partial}{\partial z}\left(n D \frac{\partial c_{f}}{\partial z}\right)-n v_{f} \frac{\partial c_{f}}{\partial z}-(1-n) v_{s} \frac{\partial c_{s}}{\partial z} .
$$

Comparing Eqs. (7) and (9), it is clear that Eq. (9) is a special case of Eq. (7) with $S_{r}=1, \beta=0$, while new terms due to the compressibility of the pore water are contained in our equation.

Using the relationship between $n$ and $n^{0}$ [20], i.e.,

$$
n=n^{0}+\left(1-n^{0}\right) \frac{\partial u}{\partial z}
$$

the equations become considerably more complex. However, our main focus is on the effect of unsaturation and compressibility of pore water. If the deformation is relatively small, the volume strain, i.e., $\partial u / \partial z$ herein is consequently small, therefore $n$ in Eq. (1) and Eq. (8) will not differ appreciably from $n^{0} \operatorname{red[10].~}$ On the other hand, although the reducing porosity can narrow the aqueous solute transport path, it tends to result in a bigger intrinsic pore water velocity and in turn the advective flux. Therefore, it is a reasonable approximation of constant $n$ employed in the following non-dimensional analysis. This approach has been used in the previous investigations $[10,12]$. Keeping temporal and spatial variations of porosity, the governing equations Eqs. (1), (2) and (8) become, respectively

$$
\begin{gathered}
S_{r} n^{0} \beta \frac{\partial p^{e}}{\partial t}+S_{r} \frac{\partial^{2} u}{\partial t \partial z}=\frac{1}{\rho_{w} g} \frac{\partial}{\partial z}\left(K \frac{\partial p^{e}}{\partial z}\right), \\
G \frac{2(1-v)}{(1-2 v)} \frac{\partial^{2} u}{\partial z^{2}}+\left(\rho_{s}-\rho_{w}\right) g \frac{\partial u}{\partial z}=\frac{\partial p^{e}}{\partial z},
\end{gathered}
$$


and

$$
\begin{aligned}
{\left[S_{r} n^{0}+\left(1-n^{0}\right) \rho_{s} K_{d}\right] \frac{\partial c_{f}}{\partial t} } & =S_{r} n^{0} D_{m} \frac{\partial^{2} c_{f}}{\partial z^{2}}-\alpha_{L} \frac{K}{\rho_{w} g} \frac{\partial p^{e}}{\partial z} \frac{\partial^{2} c_{f}}{\partial z^{2}} \\
& +\frac{\partial c_{f}}{\partial z}\left\{-\alpha_{L} S_{r} n^{0} \beta \frac{\partial p^{e}}{\partial t}-\alpha_{L} S_{r} \frac{\partial^{2} u}{\partial z \partial t}\right. \\
& +\frac{\alpha_{L} \beta K}{\rho_{w} g}\left(\frac{\partial p}{\partial z}\right)^{2}+S_{r} D_{m}\left(1-n^{0}\right) \frac{\partial^{2} u}{\partial z^{2}} \\
& \left.+\frac{K}{\rho_{w} g} \frac{\partial p^{e}}{\partial z}-\left[S_{r} n^{0}+\left(1-n^{0}\right) \rho_{s} K_{d}\right] \frac{\partial u}{\partial t}\right\} \\
& +S_{r} n \beta \frac{\partial p^{e}}{\partial t} c_{f}-\beta \frac{K}{\rho_{w} g}\left(\frac{\partial p^{e}}{\partial z}\right)^{2} c_{f} \\
& +S_{r} n^{0} \beta \frac{\partial u}{\partial t} \frac{\partial p^{e}}{\partial z} c_{f} .
\end{aligned}
$$

\subsection{Non-dimensional analysis of coupled equations}

To understand the influence of each term in the governing equations, here the variables are non-dimensionalized and the order of each term is considered with a scaled quantity relative to a characteristic unit,

$$
\begin{gathered}
p^{*}=\frac{p^{e}}{p_{c}}, t^{*}=\frac{t}{t_{c}}, z^{*}=\frac{z}{l_{c}}, u^{*}=\frac{u}{u_{c}}, c^{*}=\frac{c_{f}}{c_{0}}, \\
t_{c}=\frac{(1-2 v) L^{2} S_{r} \rho_{w} g}{2(1-v) G K}, \\
p_{c}=\frac{2(1-v) G\left[S_{r} n^{0}+\left(1-n^{0}\right) \rho_{s} K_{d}\right]}{(1-2 v) S_{r}}, \\
u_{c}=\frac{L\left[S_{r} n^{0}+\left(1-n^{0}\right) \rho_{s} K_{d}\right]}{S_{r}},
\end{gathered}
$$

where the characteristic unit for length, $l_{c}$, is the thickness of the soil layer and $c_{0}$ is the reference solute mass concentration. 
Here, $t_{c}$ is similar to the consolidation time factor $T_{v}$ in Terzaghi consolidation theory [25]. However, $t_{c}$ incorporates the degree of saturation rather than $L^{2} / c_{v}$ $\left(c_{v}=[2(1-v) G K] /\left[\rho_{w} g(1-2 v)\right]\right.$, is the coefficient of consolidation). Both $p_{c}$ (related to the soil shear modulus) and $u_{c}$ reflect the influence of unsaturation and solute retardation due to sorption.

With the coefficients $A_{i}$ given in Table 1, the non-dimensional governing equations are

$$
\begin{gathered}
A_{1} \frac{\partial p^{*}}{\partial t^{*}}+\frac{\partial^{2} u^{*}}{\partial t^{*} \partial z^{*}}=\frac{\partial^{2} p^{*}}{\partial z^{* 2}} \\
\frac{\partial^{2} u^{*}}{\partial z^{* 2}}+A_{2} \frac{\partial u^{*}}{\partial z^{*}}=\frac{\partial p^{*}}{\partial z^{*}} \\
\frac{\partial c^{*}}{\partial t^{*}}=A_{3} \frac{\partial^{2} c^{*}}{\partial z^{* 2}}-A_{4} \frac{\partial p^{*}}{\partial z^{*}} \frac{\partial^{2} c^{*}}{\partial z^{* 2}}+\frac{\partial c^{*}}{\partial z^{*}}\left(-A_{1} A_{4} \frac{\partial p^{*}}{\partial t^{*}}-A_{4} \frac{\partial^{2} u^{*}}{\partial z^{*} \partial t^{*}}+A_{5} \frac{\partial p^{*}}{\partial z^{*}} \frac{\partial p^{*}}{\partial z^{*}}\right. \\
\left.+A_{6} \frac{\partial^{2} u^{*}}{\partial z^{* 2}}+\frac{\partial p^{*}}{\partial z^{*}}-A_{7} \frac{\partial u^{*}}{\partial t^{*}}\right)+A_{1} \frac{\partial p^{*}}{\partial t^{*}} c^{*}-A_{8}\left(\frac{\partial p^{*}}{\partial z^{*}}\right)^{2} c^{*}+A_{1} A_{7} \frac{\partial u^{*}}{\partial t^{*}} \frac{\partial p^{*}}{\partial z^{*}} c^{*}
\end{gathered}
$$

We interpret the coefficients in Eqs. (18)-(20) as follows. $A_{1}$ represents the ratio of skeleton modulus to that of pore fluid, which becomes notable for an unsaturated stiff porous medium. $A_{2}$ represents the body force effect on consolidation, and is analogous to the "body force number" in [20]. When it is negligible, the effect of self-weight can be ignored. $A_{3}=\left(t_{c} S_{r}\left(n^{0}\right)^{2} D_{m}\right) /\left(\left[S_{r} n^{0}+\left(1-n^{0}\right) \rho_{s} K_{d}\right] L^{2}\right)$ can be seen as the reciprocal of Péclet number with the modifications of retardation and unsaturation. $A_{4}$ is longitudinal dispersivity per unit length. $A_{7}$ is the modified retardation factor including unsaturation. 
Table 1: Coefficients $A_{1}-A_{8}$ used in the governing equations (Eqs. (18-20))

\begin{tabular}{lll}
\hline Coefficient & Expression & Physical meaning \\
\hline$A_{1}$ & $\frac{2 G(1-v) n^{0} \beta}{1-2 v}$ & Ratio of skeleton modulus to that of pore fluid \\
$A_{2}$ & $\frac{\left(1-n^{0}\right)\left(\rho_{s}-\rho_{w}\right) g L(1-2 v)}{2 G(1-v)}$ & Body force effect on consolidation \\
$A_{3}$ & $\frac{S_{r}^{2} n^{0} D_{m} \rho_{w} g(1-2 v)}{2\left[S_{r} n^{0}+\left(1-n^{0}\right) \rho_{s} K_{d}\right] G(1-v) K}$ & Reciprocal of Péclet number with \\
$A_{4}$ & $\frac{\alpha_{L}}{L}$ & modification for retardation and unsaturation \\
$A_{5}$ & $\frac{A_{1} A_{4} A_{7}}{n^{0}}$ & \\
$A_{6}$ & $\frac{A_{3} A_{7}\left(1-n^{0}\right)}{n^{0}}$ & \\
$A_{8}$ & $\frac{S_{r} n^{0}+\left(1-n^{0}\right) \rho_{s} K_{d}}{S_{r}}$ & \\
$A_{7}$ & & \\
\hline$A^{0}$ &
\end{tabular}




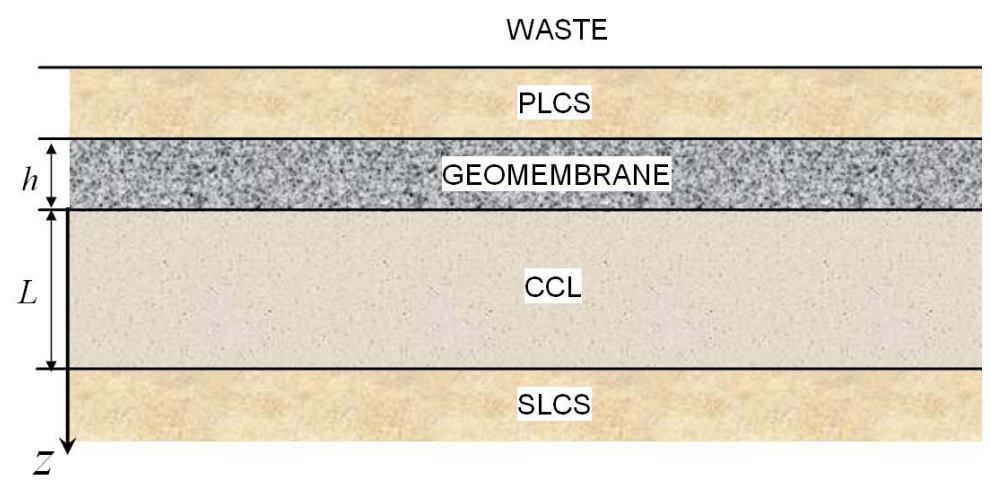

Figure 1: A schematic of an engineered landfill liner

\section{Application to a landfill profile}

The landfill liner system is similar to the operational liner investigated previously [10], as depicted in Fig. 1 It includes a primary leachate collection system (PLCS), a geomembrane overlying a compacted clay liner (CCL), and a secondary leachate collection system (SLCS) (Fig. 1). The origin of the vertical axis is located on the top of the CCL.

The landfill is assumed to fill with waste at a fixed loading rate until it reaches its capacity. redAt the top boundary, the impermeable geomembrane prevents Darcy flow, and the total vertical stress equals the surcharge loading, i.e.,

$$
q(0, t)=-\frac{K}{\rho_{w} g} \frac{\partial p^{e}(0, t)}{\partial z}=0, \quad G \frac{2(1-v)}{(1-2 v)} \frac{\partial u(0, t)}{\partial z}=-Q(t)+p^{e} .
$$

redThe volatile organic compounds diffuse through the thin (relative to CCL) geomembrane at the top boundary, and the solute flux can be approximated as

$$
f\left(0^{-}, t\right)=-D_{G} \frac{c_{f}\left(0^{+}, t\right)-c_{0}}{h},
$$

redwhile the flux in the CCL at the interface is 


$$
f\left(0^{+}, t\right)=-n D \frac{\partial c_{f}}{\partial z}\left(0^{+}, t\right)
$$

redEquating Eq. (22) and Eq. (23) [10]

$$
\frac{\partial c_{f}}{\partial z}(0, t)-\frac{D_{G}}{n\left(0^{+}, t\right) h D} c_{f}(0, t)=-\frac{D_{G}}{n\left(0^{+}, t\right) h D} c_{0} .
$$

redHere, the zero Darcy flow but non-zero contaminant solute flux makes it impossible to utilize analytical solutions. Some analytical solutions for solute transport in porous media without a geomembrane are available [26, 27, 28].

redAt the lower fixed boundary of the clay liner, the pore fluid is assumed to drain freely, and the gradient of solute concentration is assumed to be zero (Danckwert's boundary condition, [29]), although different interpretations of this condition are possible (e.g., [30]):

$$
p^{e}(L, t)=0, \quad u(L, t)=0, \quad \frac{\partial c_{f}}{\partial z}(L, t)=0 .
$$

The initial excess pore water pressure, soil displacement and solute concentration in the clay liner are zero. That is,

$$
p^{e}(z, 0)=0, u(z, 0)=0, \quad c_{f}(z, 0)=0 .
$$

\subsection{Comparison with previous work [10]}

FEM codes for various models were constructed using the multiphysics modeling software package COMSOL 3.5a [31]. These involve solution of consolidation under ramp surcharge and the solute transport equation. Since there are no models or experimental data considering the present case, it is only possible to reduce the present model to previously reported special cases (i.e., full saturation, 


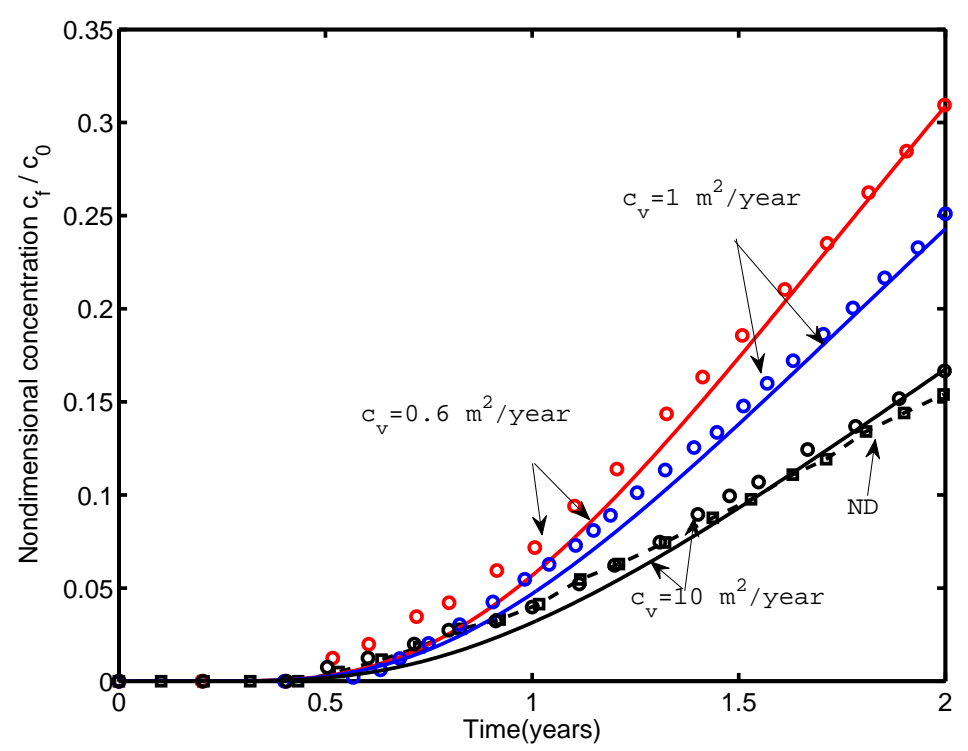

Figure 2: Comparison with previous work [10]. Present model: lines; results from [10]: circles; result of the no-deformation model (ND) from [10]: dashed line with square symbol. $L=0.914$ $\mathrm{m}, n^{0}=0.25, D_{m}=0.1 \mathrm{~m}^{2} / \mathrm{y}\left(S_{r}=1\right.$ and $\left.\beta=0\right)$.

$\left.S_{r}=1, \beta=0\right)$. When $A_{1}, A_{2}, A_{4} \sim A_{6}$ and $A_{8}$ are zero, the present model reduces to the small deformation model of [10]. In the FEM analysis, the system was discretized into unstructured Lagrange-linear elements with a maximum global element size of $10^{-2} \mathrm{~m}$, and maximum local element size at the end boundaries (where the most rapid changes occur) of $10^{-4} \mathrm{~m}$. Temporally, the sub-time step was $10^{-2}$ year. As shown in Fig. 2, the present model agrees well with earlier results [10].

\subsection{Dimensionless analysis}

In this section, we discuss the significance of each term in the governing equations. Based on the numerical examples used in [10] and [22], the input data 
or parameters adopted in the landfill clay barrier system are listed in Table 2. It should be noted that inter-relationships exist among the various $A_{i} \mathrm{~s}$. Further, $(2 G(1-v) K) /(1-2 v)$ in $A_{3}$ should be restricted to a reasonable range of $c_{v}$. Additionally, given the assumption on porosity, the choice of parameters should ensure that the non-dimensional soil deformation is relatively small, e.g., less than 20\%. Assuming $S_{r}=0.8 \sim 1.0, \alpha_{L}=0.1 \mathrm{~m}, v=0.33$, we focus on variations of $S_{r}(\beta), D_{m}, K, G$ and present the magnitude of each coefficient as in Table 3. The characteristic parameters are, $t_{c}=4.97 \times 10^{7} \mathrm{~s}(1.576 \mathrm{y}), p_{c}=6.50 \times 10^{5}$ $\mathrm{Pa}, u_{c}=0.33 \mathrm{~m}$ for case 1 and $t_{c}=3.98 \times 10^{6} \mathrm{~s}(0.126 \mathrm{y}), p_{c}=6.50 \times 10^{6} \mathrm{~Pa}$, $u_{c}=0.33 \mathrm{~m}$ for case 2 .

The corresponding non-dimensional form for the boundary conditions (BCs) and initial conditions (ICs) are

$$
\begin{gathered}
\frac{\partial p^{*}\left(0, t^{*}\right)}{\partial z^{*}}=0, \quad p^{*}\left(1, t^{*}\right)=0, \quad p^{*}\left(z^{*}, 0\right)=0 ; \\
\frac{\partial u^{*}\left(0, t^{*}\right)}{\partial z^{*}}=\frac{(1-2 v) L p_{c}}{2 G(1-v) u_{c}}\left[\frac{-Q\left(t^{*} t_{c}\right)}{p_{c}}+p^{*}\right]=\frac{-Q\left(t^{*} t_{c}\right)}{p_{c}}+p^{*}, \\
u^{*}\left(1, t^{*}\right)=0, \quad u^{*}\left(z^{*}, 0\right)=0 ; \\
\frac{\partial C^{*}\left(0, t^{*}\right)}{\partial z^{*}}=\frac{D_{G} L}{n^{0} h D_{m}}\left(C^{*}-1\right)=A_{9}\left(C^{*}-1\right), \quad \frac{\partial C^{*}\left(1, t^{*}\right)}{\partial z^{*}}=0, \quad C^{*}\left(z^{*}, 0\right)=0 .
\end{gathered}
$$

where the ratio of the mass transfer coefficients of geomembrane and clay is specified as $D_{G} / D_{m}=10^{-3}$, then $A_{9}=2.02$. 
Table 2: Typical parameter values for a landfill clay barrier system

\begin{tabular}{ll}
\hline Parameter & Value \\
\hline Waste loading, $Q(t)$ & ramp loading, $2 \times 10^{5} \mathrm{~Pa} / \mathrm{y} \times 2 \mathrm{y}$ \\
Thickness of geomembrane, $h$ & $0.0015 \mathrm{~m}$ \\
Thickness of CCL, $L$ & $0.914 \sim 1.22 \mathrm{~m}$ \\
Mass transfer coefficient of geomembrane, $D_{G}$ & $10^{-4} \mathrm{~m}^{2} / \mathrm{y}$ \\
Partitioning coefficient, $K_{d}$ & 0 \\
Effective coefficient of molecular diffusion & $5 \times 10^{-10} \sim 5 \times 10^{-9} \mathrm{~m}^{2} / \mathrm{s}$ \\
in the clay, $D_{m}$ & \\
Coefficient of consolidation in clay, $c_{v}$ & $0.6 \sim 10 \mathrm{~m}^{2} / \mathrm{y}$ \\
Shear modulus, $G$ & $5 \times 10^{5} \sim 5 \times 10^{6} \mathrm{~Pa}$ \\
Hydraulic conductivity of clay, $K$ & $10^{-10} \sim 1.5 \times 10^{-10} \mathrm{~m} / \mathrm{s}$ \\
Initial porosity of clay, $n^{0}$ & 0.33 \\
Acceleration due to gravity, $g$ & $9.8 \mathrm{~m} / \mathrm{s}^{2}$ \\
Initial density of the pore fluid, $\rho_{w}$ & $10^{3} \mathrm{~kg} / \mathrm{m}^{3}$ \\
Density of the solid phase, $\rho_{s}$ & $2.6 \times 10^{3} \mathrm{~kg} / \mathrm{m}^{3}$ \\
\hline
\end{tabular}


Table 3: Magnitude of coefficients $A_{1}-A_{8}$ used in the landfill case and simulation cases

\begin{tabular}{llll}
\hline Coefficient & Order & Case 1 & Case 2 \\
\hline$A_{1}$ & $0.13 \sim 14.05$ & 0.13 & 14.05 \\
$A_{2}$ & $5.33 \times 10^{-4} \sim 10^{-3}$ & $5.33 \times 10^{-3}$ & $5.33 \times 10^{-4}$ \\
$A_{3}$ & $1.65 \times 10^{-3} \sim 0.248$ & 0.248 & $1.99 \times 10^{-3}$ \\
$A_{4}$ & 0.1 & 0.1 & 0.1 \\
$A_{5}$ & $1.30 \times 10^{-2} \sim 1.4$ & $1.3 \times 10^{-2}$ & 1.4 \\
$A_{6}$ & $1.11 \times 10^{-3} \sim 0.166$ & 0.166 & $1.33 \times 10^{-3}$ \\
$A_{7}$ & 0.33 & 0.33 & 0.33 \\
$A_{8}$ & $0.13 \sim 14.05$ & 0.13 & 14.05 \\
\hline
\end{tabular}

\subsection{Simplification analysis}

Peters and Smith [10] and Lewis [22] performed a small deformation analysis using a spatial coordinate system. In their models [10, 22], the spatial variation of porosity, self-weight of the clay liner and longitudinal dispersivity were not considered $[10,22]$. That is because they emphasized mechanical consolidationinduced advective solute transport and the differences made by geometric and material non-linearity compared with linear models. A useful metric is the "breakthrough time", which is defined as the time for the contaminant concentration in the SLCS to reach a predetermined concentration, say 0.1 times that of concentration in landfill, i.e., $c^{*}=0.1$ in the present non-dimensional analysis.

redAt the bottom boundary, there is only an advective contaminant flux component because of the zero gradient in $c_{f}$. Besides the breakthrough time, the advective emission, redi.e., the cumulative contaminant mass outflow per unit area from the barrier system due to advective flow, is also important to evaluate the 
influence of deformation and the potential environmental risk. Provided the fixed bottom boundary, the non-dimensional advective emission can be taken as

$$
E_{a d v}^{*}=\int_{0}^{t^{*}}-\frac{\partial p^{*}(\tau)}{\partial z^{*}} c^{*}(\tau) d \tau
$$

Based on the present model, the effect of SW, SVP, CPW and longitudinal dispersion on solute transport in terms of advective solute emission at the exit boundary is examined.

For cases 1 and 2, results are given in Figs. 3 - 5, and the details for model A-G are tabulated in Table 4. As shown in Table $3, A_{2}, A_{3}$ and $A_{6}$ are relatively small. However, $A_{3}$ represents molecular diffusion mechanism, which is the main contaminant transport mechanism in the post-consolidation period. Therefore, $A_{3}$ is kept in the present model. Fig. 3 shows that the effect of SW and SVP are negligible for case 1; both can be omitted without inducing a discernible difference. Regarding case 2, the differences due to SW and SVP are even smaller because of the smaller values of $A_{3}$ and $A_{6}$ than in case 1 .

When $A_{1}=0$, the compressibility of pore water is ignored in the consolidation and transport equations. In the latter, the $\mathrm{CPW}$ gives rise to three terms similar to sources/sinks, and also terms that couple with dispersivity. Fig. 4 shows that the influence of CPW is increasingly important in case 2 (the advective emission (or flux) increases approximately four times for the case including CPW). Furthermore, results of mode $\mathrm{C}$ are close to that of the full model, which indicates that the terms arising due to $\mathrm{CPW}$ in the transport equations matter little, while the terms due to $\mathrm{CPW}$ in the consolidation equation alter the flows and dominate the emission flux. 


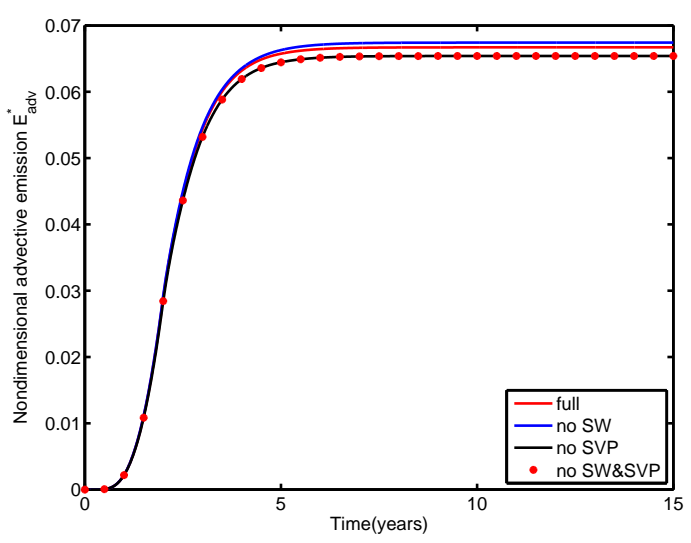

(a) Case 1

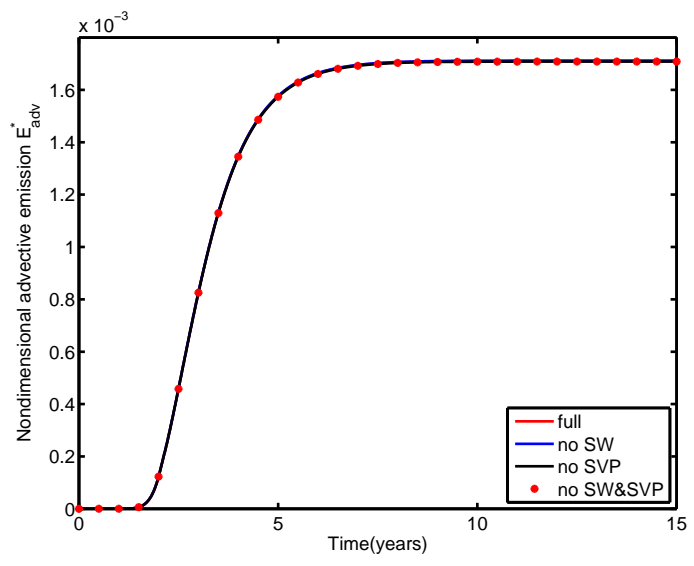

(b) Case 2

Figure 3: Influence of self-weight (SW) and spatial variation of porosity (SVP) on advective emission

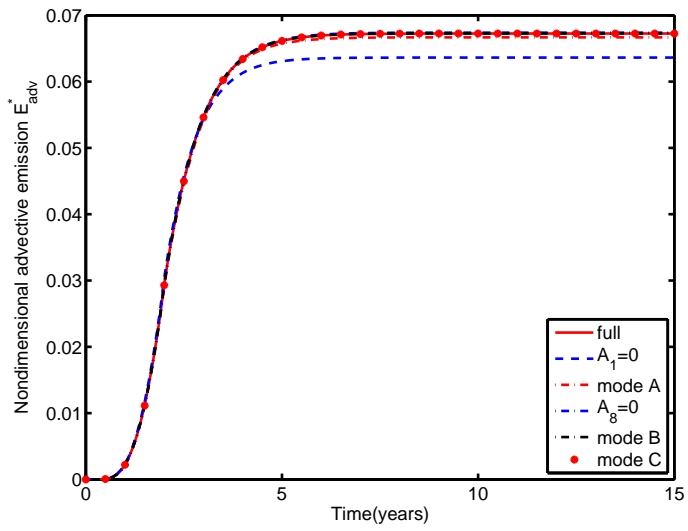

(a) Case 1

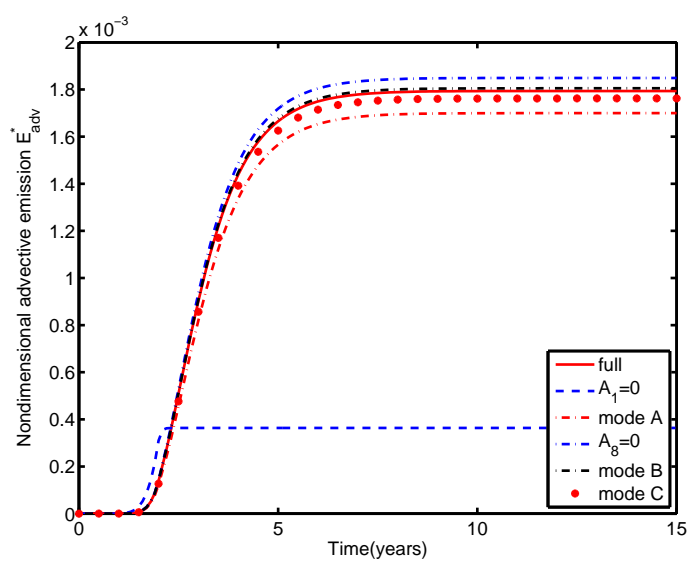

(b) Case 2

Figure 4: Influence of compressibility of pore water (CPW) on advective emission 


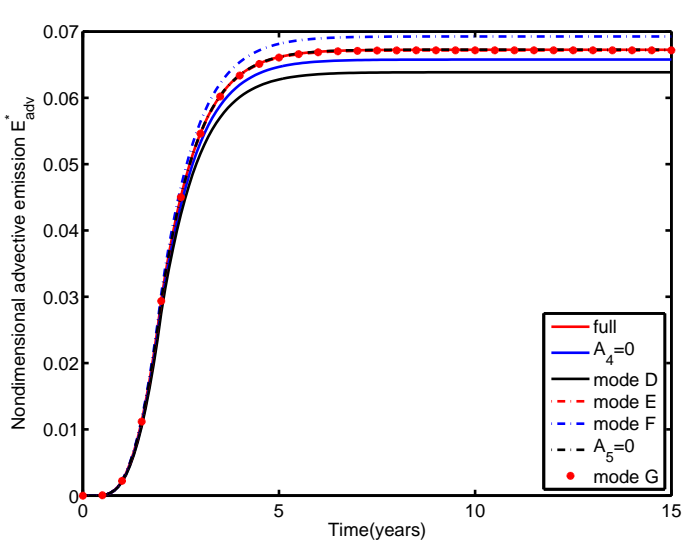

(a) Case 1

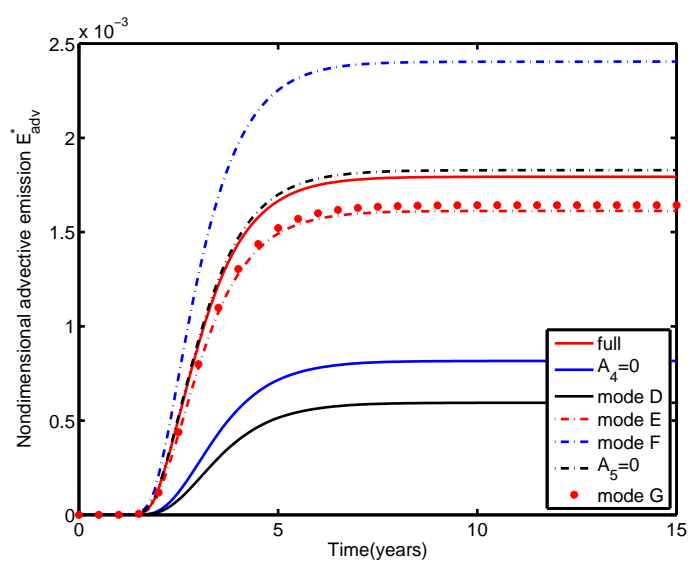

(b) Case 2

Figure 5: Influence of longitudinal dispersivity on advective emission

Table 4: Details of each model

Model Details

Mode A $A_{1} \frac{\partial p^{*}}{\partial t^{*}} c^{*}$ is omitted

Mode B $\quad A_{1} A_{7} \frac{\partial u^{*}}{\partial t^{*}} \frac{\partial p^{*}}{\partial z^{*}} c^{*}$ is omitted

Mode C $\quad A_{8}=0, A_{1} \frac{\partial p^{*}}{\partial t^{*}} c^{*}$ and $A_{1} A_{7} \frac{\partial u^{*}}{\partial t^{*}} \frac{\partial p^{*}}{\partial z^{*}} c^{*}$ are omitted

Mode $\mathrm{D} \quad A_{4} \frac{\partial p^{*}}{\partial z^{*}} \frac{\partial^{2} c^{*}}{\partial z^{* 2}}$ is omitted

Mode $\mathrm{E} \quad A_{1} A_{4} \frac{\partial p^{*}}{\partial t^{*}} \frac{\partial c^{*}}{\partial z^{*}}$ is omitted

Mode $\mathrm{F} \quad A_{4} \frac{\partial^{2} u^{*}}{\partial z^{*} \partial t^{*}} \frac{\partial c^{*}}{\partial z^{*}}$ is omitted

Mode $\mathrm{G} \quad A_{5}=0$ and $A_{1} A_{4} \frac{\partial p^{*}}{\partial t^{*}} \frac{\partial c^{*}}{\partial z^{*}}$ are omitted 
Fig. 5 shows that the effect of longitudinal dispersivity increases when $D_{m}$ decreases. In case 2 , the advective emission predicted by the model with longitudinal dispersivity is twice that of the model without longitudinal dispersivity. Among these four longitudinal dispersivity terms, the influence of $\left(\partial p^{*} / \partial z^{*}\right) \partial^{2} c^{*} / \partial z^{* 2}$ and $\left(\partial^{2} u^{*} / \partial z^{*} \partial t^{*}\right) \partial c^{*} / \partial z^{*}$ are much greater than that of $\left(\partial p^{*} / \partial t^{*}\right) \partial c^{*} / \partial z^{*}$ and $\left(\partial p^{*} / \partial z^{*}\right) \partial c^{*} / \partial z^{*}$. Therefore, it is reasonable to retain only the former two terms as in mode $\mathrm{G}$.

Based on the above analysis, the complete model can be simplified as

$$
\begin{gathered}
\frac{2 G(1-v) n \beta}{1-2 v} \frac{\partial p^{*}}{\partial t^{*}}+\frac{\partial^{2} u^{*}}{\partial t^{*} \partial z^{*}}=\frac{\partial^{2} p^{*}}{\partial z^{* 2}} \\
\frac{\partial^{2} u^{*}}{\partial z^{* 2}}=\frac{\partial p^{*}}{\partial z^{*}}, \\
\frac{\partial c^{*}}{\partial t^{*}}=\frac{S_{r}^{2} n^{0} D_{m} \rho_{w} g(1-2 v)}{2\left[S_{r} n^{0}+\left(1-n^{0}\right) \rho_{s} K_{d}\right] G(1-v) K} \frac{\partial^{2} c^{*}}{\partial z^{* 2}}-\frac{\alpha_{L}}{L} \frac{\partial p^{*}}{\partial z^{*}} \frac{\partial^{2} c^{*}}{\partial z^{* 2}} \\
+\frac{\partial c^{*}}{\partial z^{*}}\left\{-\frac{\alpha_{L}}{L} \frac{\partial^{2} u^{*}}{\partial z^{*} \partial t^{*}}+\frac{\partial p^{*}}{\partial z^{*}}-\frac{\left[S_{r} n^{0}+\left(1-n^{0}\right) \rho_{s} K_{d}\right]}{S_{r}} \frac{\partial u^{*}}{\partial t^{*}}\right\} .
\end{gathered}
$$

As shown in Fig. 6, the proposed model gives a reasonable approximation to the full model.

\subsection{Effects of degree of saturation and loading}

Based on the simplified model, the effect of degree of saturation $S_{r}$ and loading progress on the contaminant breakthrough and advective emission are examined. Fig. 7 shows that $S_{r}$ has no discernible effect on the transit time for contaminant to pass through the landfill liner. Furthermore, there is little change in the 


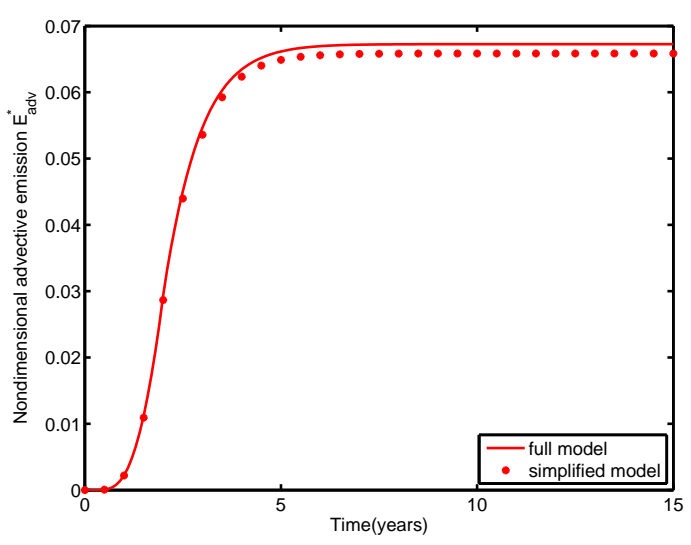

(a) Case 1

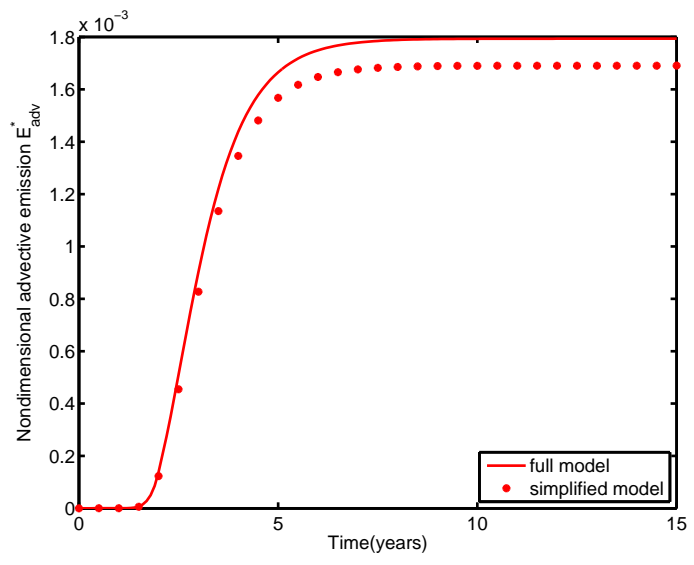

(b) Case 2

Figure 6: Comparison of advective emission between the simplified and complete full models

advective emission during the loading period. However, a lower degree of saturation leads to greater average fluid velocity, which further increases the emission flux, yielding significantly different advective emission in the post-loading period. This demonstrates the necessity of including the advective emission in estimating the effect of deformation on solute transport.

Fig. 8 shows that a larger loading rate results in smaller advective emission with the same total surcharge. Faster loading leads to faster solute transit initially, but the concentration at the exit boundary will reach the same level. Although the final water emission is the same (in Fig. 8(c)), the gap in advective emission of solute is huge, more than two orders of magnitude in the considered case. This is attributed to fluid velocities, as the fluid acts as the carrier of solute. During the early stage, greater fluid velocity of fast loading misses the gradual rise in concentration. This finding can serve as a guide for planning of loading in landfill facilities. 


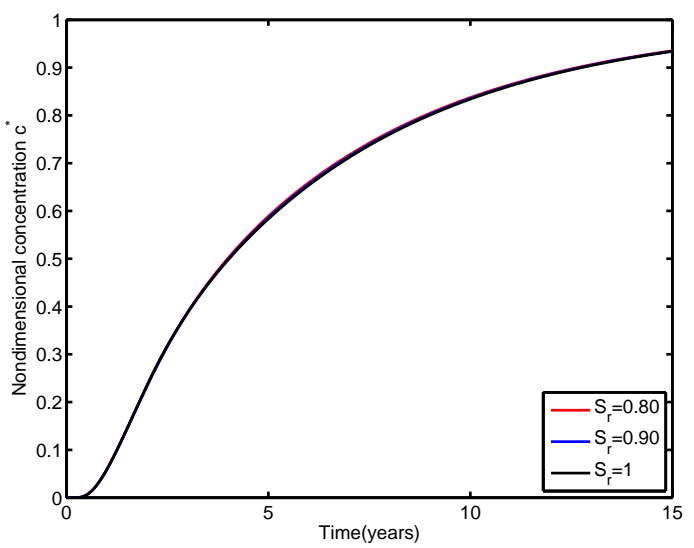

(a) $c^{*}$

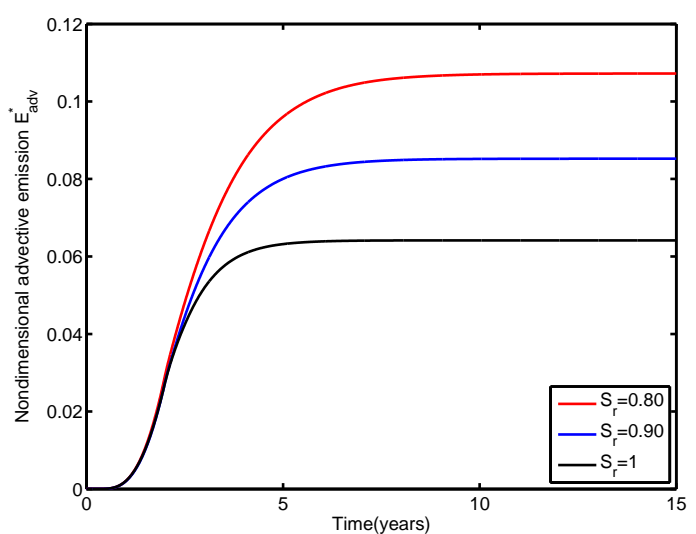

(b) $E_{a d v}^{*}$

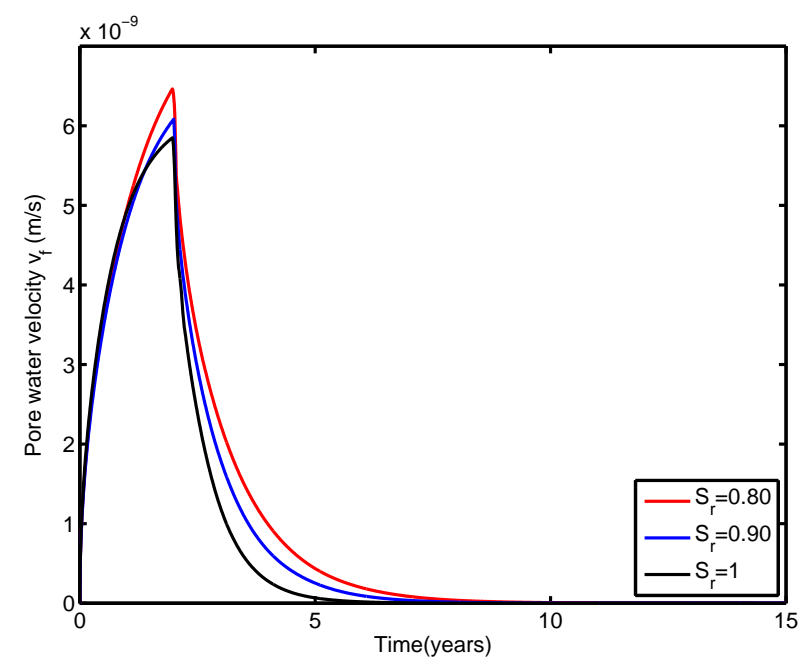

(c) $v_{f}$

Figure 7: Influence of degree of saturation $S_{r} . D_{m}=5 \times 10^{-9} \mathrm{~m}^{2} / \mathrm{s}, K=1 \times 10^{-10} \mathrm{~m} / \mathrm{s}, G=5 \times 10^{5}$ Pa. 


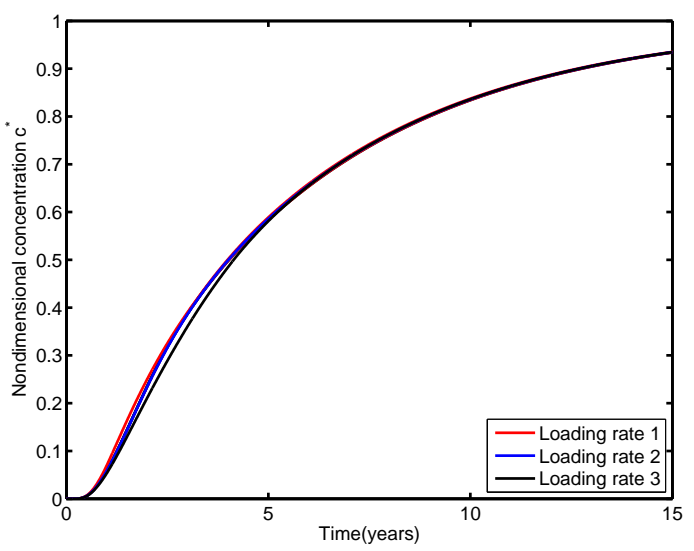

(a) $c^{*}$

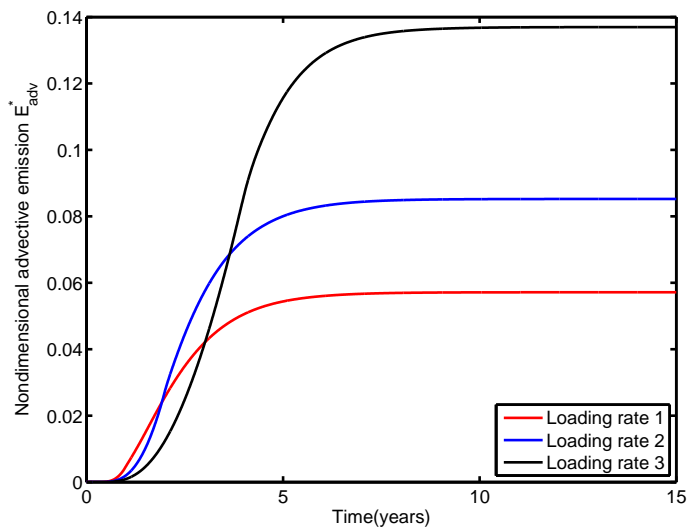

(b) $E_{a d v}^{*}$

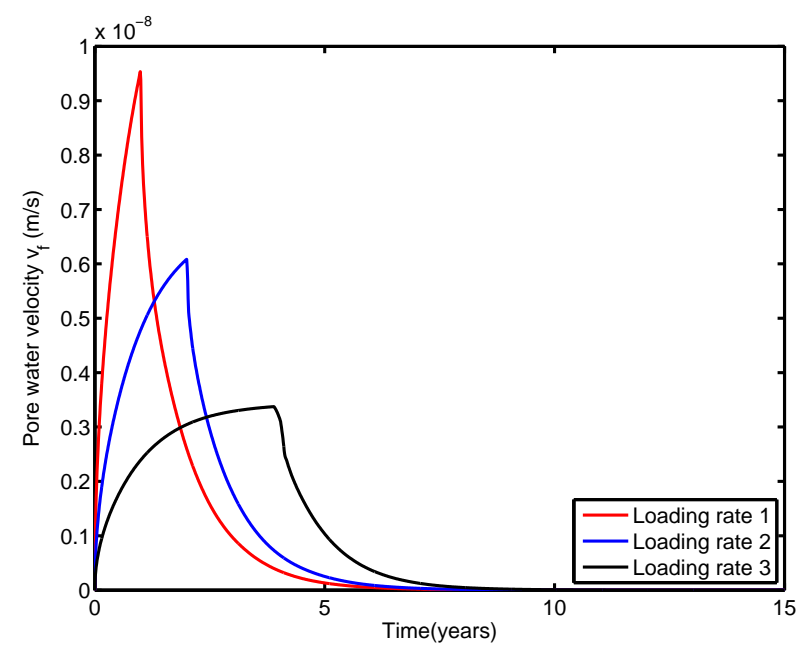

(c) $v_{f}$

Figure 8: Influence of loading process: loading rate $1 \sim 3$ represent $4 \times 10^{5} \mathrm{~Pa} / \mathrm{y}$ continuing for 1 year, $2 \times 10^{5} \mathrm{~Pa} / \mathrm{y}$ continuing for 2 year and $1 \times 10^{5} \mathrm{~Pa} / \mathrm{y}$ continuing for 4 year, respectively. $S_{r}=0.9, D_{m}=5 \times 10^{-9} \mathrm{~m}^{2} / \mathrm{s}, K=1 \times 10^{-10} \mathrm{~m} / \mathrm{s}, G=5 \times 10^{5} \mathrm{~Pa}$. 


\section{Conclusion}

In this study, the fluid storage equation and advective-diffusive equation for nearly saturated deformable porous media was proposed. Non-dimensional analysis of the comprehensive equations in the landfill liner application demonstrated that the influences of self-weight and spatial variation of porosity are negligible. However, the effects of longitudinal dispersivity and compressibility of pore water are significant in some circumstances. The parametric analysis investigated both breakthrough and advective emission of contamination, and the results indicated that the lower saturation leads to more advective emission due to greater fluid velocity, and that the slow loading rate of surcharge increases the total advective emission significantly. Furthermore, it demonstrates the advective emission should be considered besides the breakthrough curve in estimating effect of mechanical deformation on solute transport.

\section{References}

[1] J. Bear, Dynamics of Fluids in Porous Mmedia, Elsevier Scientific Publishing Company, New York, 1972.

[2] D. A. Barry, G. Sposito, Analytical solution of a convection-dispersion model with time-dependent transport coefficients, Water Resources Research 25 (1989) 2407-2416.

[3] D. A. Barry, Supercomputers and their use in modeling subsurface solute transport, Reviews of Geophysics 28 (1990) 277-295. 
[4] D. A. Barry, Modelling Chemical Transport in Soil: Natural and Applied Contaminants, Lewis Publishers, Boca Raton, Florida, USA, 1992, Ch. Modelling contaminant transport in the subsurface: Theory and computer programs, pp. 105-144.

[5] L. Li, D. A. Barry, D.-S. Jeng, Tidal fluctuations in a leaky confined aquifer: Dynamic effects of an overlying phreatic aquifer, Water Resources Research37 (4) (2001) 1095-1098.

[6] D. W. Smith, One-dimensional contaminant transport through a deforming porous medium : Theory and a solution for a quasi-steady-state problem, International Journal for Numerical and Analytical Methods in Geomechanics 24 (2000) 693-722.

[7] F. Arega, E. Hayter, Coupled consolidation and contaminant transport model for simulating migration of contaminants through the sediment and a cap, Applied Mathematical Modelling 32 (2008) 2413-2428.

[8] L. Zhang, A. Szeri, Transport of neutral solute in articular cartilage: Effects of loading and particle size, Proceedings of the royal society a-mathematical physical and engineering sciences 461 (2005) 2021-2042.

[9] L. J. Potter, C. Savvidou, R. E. Gibson, Consolidation and pollutant transport associated with slurried mineral waste disposal, in: 1st International Conference on Environmental Geotechnics, Edmonton, Canada, 1994, pp. $525-530$. 
[10] G. P. Peters, D. W. Smith, Solute transport through a deforming porous medium, International Journal for Numerical and Analytical Methods in Geomechanics 26 (2002) 683-717.

[11] H. Moo-Young, T. Myers, B. Tardy, R. Ledbetter, W. Vanadit-Ellis, T.-H. Kim, Centrifuge simulation of the consolidation characteristics of capped marine sediment beds, Engineering Geology 70 (3-4) (2003) 249 - 258, third British Geotechnical Society Geoenvironmental Engineering Conference.

[12] A. N. Alshawabkeh, N. Rahbar, T. Sheahan, A model for contaminant mass flux in capped sediment under consolidation, Journal of Contaminant Hydrology 78 (2005) 147-165.

[13] T. W. Lewis, Theoretical effects of consoldation on solute transport in soil barriers, Ph.D. thesis, The University of Newcastle, Australia (May 2009).

[14] P. J. Fox, Coupled large strain consolidation and solute transport. i: Model development, Journal of Geotechnical and Geoenvironmental Engineering, ASCE 133 (2007) 3-15.

[15] P. J. Fox, Coupled large strain consolidation and solute transport.ii: Model verification and simulation results, Journal of Geotechnical and Geoenvironmental Engineering 133 (2007) 16-29.

[16] P. J. Fox, J. Lee, Model for consolidation-induced solute transport with nonlinear and nonequilibrium sorption, International Journal of Geomechanics, ASCE 8 (2008) 188-198. 
[17] S. Fityus, D. W. Smith, J. R. Booker, Contaminant transport through an unsaturated soil liner beneath a landfill, Canadian Geotechnical Journal 36 (1999) 330-354.

[18] J. R. C. Hsu, D.-S. Jeng, C. P. Lee, Oscillatory soil response and liquefaction in an unsaturated layered seabed, International Journal for Numerical and Analytical Methods in Geomechanics 19 (12) (1995) 825-849.

[19] D.-S. Jeng, Y. S. Lin, Non-linear wave-induced response of porous seabed: A finite element analysis, International Journal for Numerical and Analytical Methods in Geomechanics 21 (1) (1997) 15-42.

[20] T.-L. Tsai, K.-C. Chang, L.-H. Huang, Body force effect on consolidation of porous elastic media due to pumping, Journal of Chinese Institute of Engineers 29 (2006) 75-82.

[21] N. Klett, T. B. Edil, C. H. Benson, J. Connelly, Evaluation of volatile organic compounds in wisconsin landfill leachate and lysimeter samples, Tech. rep., Department of Civil and Environmental Engineering, University of Wisconsin-Madison, Madison, USA (2005).

[22] T. W. Lewis, P. Pivonka, D. W. Smith, Theoretical investigation of the effects of consolidation on contaminant transport through clay barriers, International Journal For Numerical And Analytical Methods In Geomechanics 33 (2008) 95-116.

[23] J. Bear, A. H.-D. Cheng, Modelling groundwater flow and contaminant transport, Springer, Heidelberg, 2009. 
[24] D. Fredlund, H. Rahardjo, Soil mechanics for unsaturated soils, WileyInterscience, New York, 1993.

[25] K. Terzaghi, Erdbaumechanik auf Bodenphysikalischer Grundlage, F. Deuticke, Vienna, Deuticke, 1925.

[26] R. K. Rowe, M. ASCE, J. R. Booker, 1-d pollutant migration in soil of finite depth, Journal of Geotechnical Engineering 111 (1985) 479-499.

[27] J. Guerrero, T. Skaggs, Analytical solution for one-dimensional advectiondispersion transport equation with distance-dependent coefficients, Journal of Hydrology 390 (1-2) (2010) 57-65.

[28] Y.-C. Li, P. J. Cleall, Analytical solutions for advectivedispersive solute transport in double-layered finite porous media, International Journal for $\mathrm{Nu}$ merical and Analytical Methods in Geomechanics 35 (4) (2011) 438-460.

[29] P. V. Danckwerts, Continuous flow systems : Distribution of residence times, Chemical Engineering Science 2 (1953) 1-13.

[30] D. A. Barry, G. Sposito, Application of the convection-dispersion model to solute transport in finite soil columns, Soil Science Society of America Journal 52 (1988) 3-9.

[31] COMSOL Multiphysics, 3rd Edition (2010).

[32] A. Verruijt, Elastic storage of aquifers, (edited by R J M De Wiest) Academic Press, New York, 1969, Ch. 8, pp. 331-376. 


\section{Appendix: Derivation of fluid storage and solute transport equations}

The macroscopic mass conservative equation for pore water in a general form is [23]

$$
\frac{\partial}{\partial t}\left(\theta \rho_{w}\right)=-\nabla \cdot\left(\rho_{w} \theta \overrightarrow{\mathbf{v}_{f}}\right)
$$

where the volume fraction $\theta$ is related to porosity $n$ and degree of saturation $S_{r}$ by $\theta=S_{r} n, \rho_{w}$ is density of pore water, $\overrightarrow{\mathbf{v}}_{f}$ denotes the average fluid velocity vector, which can be related to specific discharge relative to solid, $\overrightarrow{\mathbf{q}}_{r}$ based on Darcy's law by

$$
\overrightarrow{\mathbf{q}_{r}}=\theta\left(\overrightarrow{\mathbf{v}_{f}}-\overrightarrow{\mathbf{v}}_{s}\right)=-\frac{K}{\rho_{w} g} \nabla p^{e},
$$

where $\overrightarrow{\mathbf{v}}_{s}$ is velocity of the solid.

Assuming $\rho_{w}$ depends only on $p$, and with the definition of coefficient of compressibility $\beta\left(=\left(1 / \rho_{w}\right) d \rho_{w} / d p\right)$, Eq. (34) yields

$$
\frac{\partial S_{r} n}{\partial t}+\nabla \cdot\left(S_{r} n \overrightarrow{\mathbf{v}}_{f}\right)=-S_{r} n \beta\left(\frac{\partial p^{e}}{\partial t}+\overrightarrow{\mathbf{v}}_{f} \cdot \nabla p^{e}\right)
$$

In view of Darcy's law, it becomes

$$
\frac{\partial S_{r} n}{\partial t}+S_{r} \nabla \cdot\left(n \overrightarrow{\mathbf{v}}_{s}\right)-\nabla \cdot\left(\frac{K}{\rho_{w} g} \nabla p^{e}\right)=-S_{r} n \beta\left(\frac{\partial p^{e}}{\partial t}+\overrightarrow{\mathbf{v}}_{f} \cdot \nabla p^{e}\right)
$$

Using the chain rule, we have

$$
\nabla \cdot\left(\frac{K}{\rho_{w} g} \nabla p^{e}\right)=\frac{1}{\rho_{w} g} \nabla \cdot\left(K \nabla p^{e}\right)-\frac{K}{\rho_{w} g} \beta \nabla p^{e} \cdot \nabla p^{e} .
$$

Substituting this expression and Eq. (35) into Eq. (37) gives 


$$
\begin{aligned}
\frac{\partial S_{r} n}{\partial t}+S_{r} \nabla \cdot\left(n \overrightarrow{\mathbf{v}_{s}}\right)-\frac{1}{\rho_{w} g} \nabla \cdot\left(K \nabla p^{e}\right) & -S_{r} n \beta\left(\overrightarrow{\mathbf{v}}_{f}-\overrightarrow{\mathbf{v}}_{s}\right) \cdot \nabla p^{e} \\
& =-S_{r} n \beta\left(\frac{\partial p^{e}}{\partial t}+\overrightarrow{\mathbf{v}}_{f} \cdot \nabla p^{e}\right) .
\end{aligned}
$$

Rearranging,

$$
\frac{\partial S_{r} n}{\partial t}+S_{r} \nabla \cdot\left(n \overrightarrow{\mathbf{v}_{s}}\right)-\frac{1}{\rho_{w} g} \nabla \cdot\left(K \nabla p^{e}\right)=-S_{r} n \beta\left(\frac{\partial p^{e}}{\partial t}+\overrightarrow{\mathbf{v}}_{s} \cdot \nabla p^{e}\right)
$$

Regarding the solid phase, its mass conservation equation is given by

$$
\frac{\partial}{\partial t}\left[(1-n) \rho_{s}\right]=-\nabla \cdot\left((1-n) \rho_{s} \overrightarrow{\mathbf{v}}_{s}\right) .
$$

Since the deformation modulus of soil particles is relatively large under usual loading, deformation of the solid phase is assumed to be volume preserving, i.e., $D_{s} \rho_{s} / D t=0$ (where $D_{s} / D t$ is the material derivation), hence,

$$
\frac{\partial n}{\partial t}=(1-n) \nabla \cdot \overrightarrow{\mathbf{v}}_{s}-\overrightarrow{\mathbf{v}}_{s} \cdot \nabla n=\nabla \cdot \overrightarrow{\mathbf{v}}_{s}-\nabla \cdot\left(n \overrightarrow{\mathbf{v}_{s}}\right) .
$$

Therefore, Eq. (40) becomes

$$
S_{r} \nabla \cdot \overrightarrow{\mathbf{v}}_{s}-\frac{1}{\rho_{w} g} \nabla \cdot\left(K \nabla p^{e}\right)=-S_{r} n \beta\left(\frac{\partial p^{e}}{\partial t}+\overrightarrow{\mathbf{v}}_{s} \cdot \nabla p^{e}\right) .
$$

In case of relatively small deformations, it is reasonable to make the assumption that the advective component variation of $p^{e}$ can be ignored [23], i.e.,

$$
\left|\frac{\partial p^{e}}{\partial t}\right| \gg\left|\overrightarrow{\mathbf{v}_{s}} \cdot \nabla p^{e}\right|
$$

thus, 


$$
S_{r} n \beta \frac{\partial p^{e}}{\partial t}+S_{r} \nabla \cdot \overrightarrow{\mathbf{v}}_{s}=\frac{1}{\rho_{w} g} \nabla \cdot\left(K \nabla p^{e}\right) .
$$

When $S_{r}=1$, it leads to

$$
n \beta \frac{\partial p^{e}}{\partial t}+\nabla \cdot \overrightarrow{\mathbf{v}}_{s}=\frac{1}{\rho_{w} g} \nabla \cdot\left(K \nabla p^{e}\right),
$$

which is equivalent to the well-known storage equation of [32].

Consideration of solute mass in the fluid phase for a fixed representative element volume (REV) leads to

$$
\frac{\partial\left(n S_{r} c_{f}\right)}{\partial t}+s=-\frac{\partial}{\partial z}\left[n S_{r}\left(-D \frac{\partial c_{f}}{\partial z}+v_{f} c_{f}\right)\right]
$$

where, $s$ is the rate of solute mass source per unit volume. The rate of solute loss by sorption onto the solid phase is equal to the rate of the solute gain by the solid phase from the fluid phase. It is noted that the amount and identity of matter in the REV may change with time, while the shape and position of this volume remain fixed. However, for the deformable porous medium considered in this study, the porosity $n$ is time-dependent, and the macroscopic velocity of the solid matrix is not zero.

Conservation of solute mass for the solid phase is given by

$$
\frac{\partial\left[(1-n) c_{s}\right]}{\partial t}-s=-\frac{\partial}{\partial z}\left[(1-n) v_{s} c_{s}\right] .
$$

Finally, the transport equation for a solute in a deforming porous medium is expressed by combination of Eqs. (47) and (48),

$$
\frac{\partial\left(n S_{r} c_{f}\right)}{\partial t}+\frac{\partial\left[(1-n) c_{s}\right]}{\partial t}=-\frac{\partial}{\partial z}\left[n S_{r}\left(-D \frac{\partial c_{f}}{\partial z}+v_{f} c_{f}\right)+(1-n) v_{s} c_{s}\right]
$$


By the use of the mass balance equations for the fluid phase (Eq. (36)), and solid phases (Eq. (41)), and keeping in mind that $\rho_{s}$ is constant, Eq. (49) can be simplified to

$$
\begin{aligned}
S_{r} n \frac{\partial c_{f}}{\partial t}+(1-n) \frac{\partial c_{s}}{\partial t}= & \frac{\partial}{\partial z}\left(S_{r} n D \frac{\partial c_{f}}{\partial z}\right)-S_{r} n v_{f} \frac{\partial c_{f}}{\partial z} \\
& -(1-n) v_{s} \frac{\partial c_{s}}{\partial z}+S_{r} n \beta\left(\frac{\partial p^{e}}{\partial t}+v_{f} \frac{\partial p^{e}}{\partial z}\right) c_{f} .
\end{aligned}
$$

Assuming the linear sorption relationship as described by Eq. (5) and (6), expanding Eq. (50) leads to

$$
\begin{aligned}
{\left[S_{r} n+(1-n) \rho_{s} K_{d}\right] \frac{\partial c_{f}}{\partial t} } & =S_{r} n D \frac{\partial^{2} c_{f}}{\partial z^{2}}+\frac{\partial c_{f}}{\partial z}\left(S_{r} n \frac{\partial D}{\partial z}+S_{r} D \frac{\partial n}{\partial z}+\frac{K}{\rho_{w} g} \frac{\partial p^{e}}{\partial z}\right. \\
& \left.-\left[S_{r} n+(1-n) \rho_{s} K_{d}\right] \frac{\partial u}{\partial t}\right)+S_{r} n \beta \frac{\partial p^{e}}{\partial t} c_{f} \\
& -\beta \frac{K}{\rho_{w} g}\left(\frac{\partial p^{e}}{\partial z}\right)^{2} c_{f}+S_{r} n \beta \frac{\partial u}{\partial t} \frac{\partial p^{e}}{\partial z} c_{f}
\end{aligned}
$$

It is noted that the spatial derivative of porosity exists in Eq. (51). Volume conservation of the solid phase in soil can be utilized to develop its expression. For an incompressible solid:

$$
U_{s}=U_{m}(1-n)=\text { constant }
$$

where $U_{s}, U_{m}$ denote solid particle volume and the porous medium volume in the representive volume element, respectively. We separate $U_{m}$ into the initial volume $U_{m 0}$ and incremental volume $U_{m}^{e}$, Eq. (52) becomes

$$
U_{s}=\left(U_{m 0}+U_{m}^{e}\right)(1-n)
$$


By definition, volumetric strain $\varepsilon_{v}=U_{m}^{e} / U_{m 0}$. Dividing both sides of Eq. (53) by $U_{m 0}$ obtains

$$
1-n^{0}=\left(1+\varepsilon_{v}\right)(1-n)
$$

Therefore,

$$
\nabla\left(\left(1+\varepsilon_{v}\right)(1-n)\right)=0 .
$$

Correspondingly,

$$
\nabla n=\frac{(1-n)}{1+\varepsilon_{v}} \nabla \varepsilon_{v}=\frac{(1-n)^{2}}{1-n^{0}} \nabla \varepsilon_{v} .
$$

In the analysis of Peters and Smith [10], the spatial variation of $n$ was neglected. Here, this can be accommodated by use of Eq. (56). Therefore, Eq. (51) becomes

$$
\begin{aligned}
{\left[S_{r} n+(1-n) \rho_{s} K_{d}\right] \frac{\partial c_{f}}{\partial t} } & =S_{r} n D \frac{\partial^{2} c_{f}}{\partial z^{2}}+\frac{\partial c_{f}}{\partial z}\left(S_{r} n \frac{\partial D}{\partial z}+S_{r} D \frac{(1-n)^{2}}{1-n^{0}} \frac{\partial^{2} u}{\partial z^{2}}\right. \\
& \left.+\frac{K}{\rho_{w} g} \frac{\partial p^{e}}{\partial z}-\left[S_{r} n+(1-n) \rho_{s} K_{d}\right] \frac{\partial u}{\partial t}\right) \\
& +S_{r} n \beta \frac{\partial p^{e}}{\partial t} c_{f}-\beta \frac{K}{\rho_{w} g}\left(\frac{\partial p^{e}}{\partial z}\right)^{2} c_{f}+S_{r} n \beta \frac{\partial u}{\partial t} \frac{\partial p^{e}}{\partial z} c_{f}
\end{aligned}
$$

Taking into account the constant longitudinal dispersivity, Eq. (57) changes to 


$$
\begin{aligned}
{\left[S_{r} n+(1-n) \rho_{s} K_{d}\right] \frac{\partial c_{f}}{\partial t} } & =S_{r} n D_{m} \frac{\partial^{2} c_{f}}{\partial z^{2}}+S_{r} n \alpha_{L}\left(v_{f}-v_{s}\right) \frac{\partial^{2} c_{f}}{\partial z^{2}} \\
& +\frac{\partial c_{f}}{\partial z}\left\{S_{r} \alpha_{L} n \frac{\partial\left(v_{f}-v_{s}\right)}{\partial z}+S_{r} D_{m} \frac{(1-n)^{2}}{1-n^{0}} \frac{\partial^{2} u}{\partial z^{2}}\right. \\
& +S_{r} \alpha_{L}\left(v_{f}-v_{s}\right) \frac{\partial n}{\partial z}+\frac{K}{\rho_{w} g} \frac{\partial p^{e}}{\partial z} \\
& \left.-\left[S_{r} n+(1-n) \rho_{s} K_{d}\right] \frac{\partial u}{\partial t}\right\} \\
& +S_{r} n \beta \frac{\partial p^{e}}{\partial t} c_{f}-\beta \frac{K}{\rho_{w} g}\left(\frac{\partial p^{e}}{\partial z}\right)^{2} c_{f}+S_{r} n \beta \frac{\partial u}{\partial t} \frac{\partial p^{e}}{\partial z} c_{f} .
\end{aligned}
$$

Recall the chain rule,

$$
\begin{aligned}
S_{r} \alpha_{L} n \frac{\partial\left(v_{f}-v_{s}\right)}{\partial z}+S_{r} \alpha_{L}\left(v_{f}-v_{s}\right) \frac{\partial n}{\partial z} & =\alpha_{L} \frac{\partial\left[S_{r} n\left(v_{f}-v_{s}\right)\right]}{\partial z} \\
& =-\alpha_{L} \frac{\partial\left(\frac{K}{\rho_{w} g} \frac{\partial p^{e}}{\partial z}\right)}{\partial z}
\end{aligned}
$$

and employing Eq. (38) and Eq. (45), Eq. (58) becomes

$$
\begin{aligned}
{\left[S_{r} n+(1-n) \rho_{s} K_{d}\right] \frac{\partial c_{f}}{\partial t} } & =S_{r} n D_{m} \frac{\partial^{2} c_{f}}{\partial z^{2}}-\alpha_{L} \frac{K}{\rho_{w} g} \frac{\partial p^{e}}{\partial z} \frac{\partial^{2} c_{f}}{\partial z^{2}} \\
& +\frac{\partial c_{f}}{\partial z}\left\{-\alpha_{L} S_{r} n \beta \frac{\partial p^{e}}{\partial t}-\alpha_{L} S_{r} \frac{\partial^{2} u}{\partial z \partial t}\right. \\
& +\frac{\alpha_{L} \beta K}{\rho_{w} g}\left(\frac{\partial p^{e}}{\partial z}\right)^{2}+S_{r} D_{m} \frac{(1-n)^{2}}{1-n^{0}} \frac{\partial^{2} u}{\partial z^{2}} \\
& \left.+\frac{K}{\rho_{w} g} \frac{\partial p^{e}}{\partial z}-\left[S_{r} n+(1-n) \rho_{s} K_{d}\right] \frac{\partial u}{\partial t}\right\} \\
& +S_{r} n \beta \frac{\partial p^{e}}{\partial t} c_{f}-\beta \frac{K}{\rho_{w} g}\left(\frac{\partial p^{e}}{\partial z}\right)^{2} c_{f}+S_{r} n \beta \frac{\partial u}{\partial t} \frac{\partial p^{e}}{\partial z} c_{f} .
\end{aligned}
$$




\section{List of symbols}

$A_{i}$, coefficients in dimensionless equations

$c^{*}$, non-dimensional mass concentration of the solute in the fluid phase

$c_{0}$, the reference solute mass concentration, $\mathrm{ML}^{-3}$

$c_{f}$, concentration of the solute in the fluid phase, $\mathrm{ML}^{-3}$

$c_{s}$, concentration of the solute in the solid phase, $\mathrm{ML}^{-3}$

$c_{v}$, coefficient of consolidation, $\mathrm{L}^{2} \mathrm{~T}^{-1}$

$D$, hydrodynamic dispersion coefficient, $\mathrm{L}^{2} \mathrm{~T}^{-1}$

$D_{G}$, mass transfer coefficient of geomembrane, $\mathrm{L}^{2} \mathrm{~T}^{-1}$

$D_{m}$, effective molecular diffusion coefficient, $\mathrm{L}^{2} \mathrm{~T}^{-1}$

$F$, function to relate $S$ and $c_{f}$

$G$, shear modulus of soil, $\mathrm{ML}^{-1} \mathrm{~T}^{-2}$

$g$, gravity acceleration, $\mathrm{LT}^{-2}$

$h$, thickness of geomembrane, $\mathrm{L}$

$K$, hydraulic conductivity, $\mathrm{LT}^{-1}$

$K_{d}$, contaminant partitioning coefficient, $\mathrm{L}^{3} \mathrm{M}^{-1}$

$K_{w 0}$, pore water bulk modulus, $\mathrm{ML}^{-1} \mathrm{~T}^{-2}$

$L$, thickness of CCL, $\mathrm{L}$

$l_{c}$, characteristic unit for length, $\mathrm{L}$

$n$, current soil porosity

$n^{0}$, initial soil porosity

$P_{a}$, gauge air pressure, $\mathrm{ML}^{-1} \mathrm{~T}^{-2}$

$P_{0}$, atmosphere air pressure, $\mathrm{ML}^{-1} \mathrm{~T}^{-2}$

$p^{*}$, non-dimensional excess pore water pressure

$p_{c}$, characteristic unit of excess pore pressure, $\mathrm{ML}^{-1} \mathrm{~T}^{-2}$ 
$p^{e}$, excess pore pressure, $\mathrm{ML}^{-1} \mathrm{~T}^{-2}$

$r_{h}$, volumetric fraction of dissolved air

$S$, mass of contaminant sorbed onto the solid phase per unit mass of solid phase

$S_{r}$, degree of saturation

$T_{v}$, consolidation time factor in Terzaghi consolidation theory, $\mathrm{T}$

$t$, time, $\mathrm{T}$

$t^{*}$, non-dimensional time

$t_{c}$, characteristic unit for time, $\mathrm{T}$

$u$, soil displacement, $\mathrm{L}$

$u^{*}$, non-dimensional soil displacement

$u_{c}$, characteristic unit for soil displacement, $\mathrm{L}$

$v_{f}$, average fluid velocity, $\mathrm{LT}^{-1}$

$v_{s}$, solid velocity, $\mathrm{LT}^{-1}$

$z$, vertical coordinate, $\mathrm{L}$

$z^{*}$, non-dimensional vertical coordinate

\section{Greek symbols}

$\rho_{w}$, density of pore water, $\mathrm{ML}^{-3}$

$\rho_{s}$, density of soil gain, $\mathrm{ML}^{-3}$

$\beta$, compressibility of pore water, $\mathrm{LT}^{2} \mathrm{M}^{-1}$

$v$, Poisson's ratio

$\alpha_{L}$, longitudinal dispersion, $\mathrm{L}$ 